\section{The Choice of Treatment.}

In determining the variety of treatment to be employed it was necessary to discover where the disease was localized, and its type and degree of intensity. More than one examination was usually necessary for this purpose-a fact now recognized by the Ministry of Health, but, unfortunately, not by the average patient. Detailed knowledge of the past history and the present symptoms, especially those indicating toxaemia, such as rapid pulse and lowered blood pressure, should be obtained, and the examination of the temperature ought to be spread over several days, and be made at different times during the day. The sputum examination was not.the sole criterion of activity, and in some ways was even fallacious-for instance, there might be advanced disease without tubercle bacilli being found in the sputum. The $x$-ray examination was of great value in confirming the presence of tuberculosis and in defining its extent and the presence of complications. Information was also afforded as to progress during and after treatment. Tuberculin reactions had some value; the complement fixation test was still on its trial, but correct opsonic determinations were not usually obtainable; so that the decision had to be made very often on a balance of probabilities. The first point to settle was where the treatment should take place, after which the nature of the treatment could be laid down. Home treatment might be the most suitable in the febrile stage, if the conditions were good and the general practitioner understood the special requirements. After the disappearance of toxaemic symptoms the patient should go to a sanatorium. In advanced cases also home treatment was the best if the risks were recognized: it was more generally used in America than in England. One important precaution was necessary in home treatment - the patient should be made to understand that he was a patient, and should not work too much whilst waiting for sanatorium treatment. There was a great need of inexpensive nursing homes where such patients might be accommodated. With regard to the sanatorium there were many misconceptions, but Dr. Young maintained that in his opinion it was the most valuable of all the methods of treatment, though not to be considered as a standard to be applied in all stages of disease. The best type of patient for this treatment was in the early apyrexial or the old arrested stage, in which a slight breakdown had occurred. Patients in whom a successful pneumothorax operation had been performed were also suitable for sanatorium treatment. Unsuitable cases included those in which there was active and spreading disease, severe intoxication, chronic arthritis or other complications, and old age. Strength of character was a great factor in recovery, and sanatorium treatment sometimes failed because patients of weak character were turned into chronic valetudinarians. Diet was also a very important matter, and a regular weekly sequence of menus was most inadvisable. Climatic treatment had always been rery popular, except during the war period, and there were four locations to be considered-namely, the mountain, marine, inland, and desert. The great advantages of mountain treatment included the prevalent dry and dustless air. Davos and Arosa were justly famed; St. Moritz was less to be recommended for invalids nowadays. Of the lower heights Montana, Leysin, and Les Avants were frequently good for patients in whom the diagnosis was doubtful, since they could obtain treatment there without any stigma being inflicted. The followng types of the disease should not be sent to mountains for treatment: advanced cases and those with complications, especially haemoptysis, laryngitis, albuminuria, and cardiac disease. It was often advisable to spend a second winter under these conditions to complete the cure. For the second variety of climatic treatment the sea coasts were by no means unsuitable, as had once been thought, especially if the residence was a short distance inland. France and the Italian Riviera were not at all suitable for cases requiring treatment. Nowadays sea voyages were only recommended for arrested or quiescent disease. There were numerous valuable inland resorts for tuberculosis treatment, and as regards deserts the Egyptian, except in certain places, such as Helouan, was not ideal because of the absence of facilities for special treatment and the amount of dust present.

\section{VITAMINS.}

\section{MEDICAL RESEARCH COUNCIL'S REPORT.}

ThE Medical Research Council has just issued a second edition of its report on Accessory Food Factors (Vitamins). ${ }^{1}$ The first edition was published in April, 1820, at a time when the importance of vitamins was by no means universally recognized, and when many were sceptical of the claims made. But time has served to diminish the number of the sceptics and to add to the number of those who see in the study of accessory food factors and of faulty food in general a hopeful sign that the stronghold of disease has begun tc be attacked at its foundations. The gathering together intc one volume of the observations and experiments which: formed the basis of the statements made in the first edition has had much to do with the spread of knowledge in regard to these essential constituents of the food among members of the profession in general. The report served also as a source of authoritative information and of inspiration to many of those who were devoting themselves to the investigation of problems relating to vitamins. It has become the standard reference book for workers on this subject in nearly every civilized country. It is well, therefore, that the information it provides should not lag behind the times, and that it should continue to fulfil its function of furnishing a well considered account of the essential facts concerning accessory food factors so far as they are at present known. This function is admirably fulfilled in the second edition, which gives a comprehensive account of the subject, including the results of the large amount of work which has been done since the first edition was published.

There are nine chapters in the present volume, as well as a fairly complete bibliography and a convenient and well arranged index.

The first chapter, introductory to the rest, is by no means the least important, referring as it does succinctly both to what has been accomplished and to much that remains to be done. In it we are assured that although the precise chemical nature of vitamins is still unknown, their realit: has been fully established and a great number of concrete facts ascertained concerning their origin, distribution, and general physical and chemical properties. We are assured also that without these accessory factors in nutrition, which, so far as is known, cannot be synthesized in the animal, and for the supply of which it is mainly dependent on the tissues of plants, normal metabolism is not possible; while with the vitamin supply secured it seems to be proved that all known tissue constituents can be synthesized from the amino-acids of protein with the other basal foodstuffs. Surely it would be difficult to conceive of factors in nutrition more important than these, which quantitatively are infinitesimal in amount as compared with other essential constituents of the food.

\section{Vitamins and Grouth.}

The second chapter deals with accessory factors and growth. It reiterates the indispensability of the two accessory factors-fat-soluble $A$ and water-soluble $B$-for growth and nutrition, giving the observations and experiments in proof of this. The antiscorbutic substance (vitamin C), also indispensable for normal growth and nutrition, is dealt with separately in Chapter V. Of special interest are the illustrations of the influence of vitamin $A$ and vita$\min B$ on the growth and nutrition of rats, given in the second chapter; they are of fundamental importance. Young rats, when fed on an artificial ration of purified protein, carbohydrates, fat, and inorganic salts, completely fail to grow, but when the artificial ration is supplemented with adequate amounts of vitamins they, remain in excellent health, normal in growth and appearance, normal in their breeding propensities and in their capacity to rear their young, and show a normal standard of resistance to disease. In young rats the deleterious effects of deficiency of vitamin $A$ are made manifest by the appearance of keratomalacia, or by a greatly lowered state of general health, resulting in an abnormally high death rate from

1 H.M. Stationery Office, London, or through any bookseller. 1924 Pp. 171. Price 4s. 6d. 
acute infections, particularly of the lungs. The deleterious effect of vitamin $B$ deficiency in rats is evidenced by muscular inco-ordination due to involvement of the nervous system. and by serious disturbance of general health. No reference is made in this chapter to the important effect of insufficiency of this vitamin in disturbing gastro-intestinal function in these animals, although the matter is referred to in connexion with pigeons and monkeys in Chapter IV. Where both vitamin $\mathbf{A}$ and vitamin $\mathbf{B}$ are deficient in the food the deficiency of the second is that which first exerts its deleterious effect on the animal, since there is little store of it in the body for emergencies.

\section{Sources of Vitamin A.}

Chapter III deals with the distribution of vitamin A (fat-soluble $\mathbf{A}$ ) in natural foodstuffs. The valuable table in which the nutritive values of the foodstuffs as sources of this vitamin are indicated by the rough, but sufficiently accurate, method of positive and negative signs has been revised. It will be seen from it that green leaves in which active assimilation is taking place are the chief source of the vitamin; fruits, tubers, roots, and seeds containing comparatively little of it. In the animal the liver is the richest source, while the fat secreted in the milk and in eggs holds an intermediate place. The important assurance is given that there is no foundation for the belief that dark-coloured cod-liver oils contain more of the vitamin A than the white medicinal oil. Very important also is the reminder that the amount of the vitamin present in milk varies with the diet of the animal, and that animals deprived of this factor are unable to rear their young for more than a limited period; hence the need of butter fat or green leafy vegetables for nursing mothers.

This chapter deals also with the origin and physiological significance of vitamin $A$, with its properties, and with attempts to isolate it. We are told that it is synthesized by the plant; that its production, although dependent on light, is independent of carbon dioxide, oxygen, and chlorophyll; that nothing definite is known of the part it plays in nutrition of the plant or animal-although in the latter its physiological function appears to be related to the metabolism of fats, calcium, and phosphorus; that considerable amounts of it may be stored in the animal body, perhaps with the reserve fat supplies; and that different species of animals differ in their need for it. Except that young and growing animals require more and store more than fully grown animals, little appears to be known of possible variations in the needs of different animals of the same age and species-an important matter concerning which information is required.

In this chapter also the properties of vitamin $\mathbf{A}$ are discussed at length, and much that is new is to be found in regard to them in this interesting record of progress, notably in regard to the effects of oxidation in reducing the vitamin value of foodstuffs. Reference is made to the origin and source of the large stores of vitamin $A$ in the liver of the cod and many other fish. Like mammals, fish probably derive the whole of their vitamin A either directly or indirectly from the vegetable kingdom. Investigation has shown that a minute diatom (Nitzschia closterium W. Sm.) is capable of synthesizing the vitamin when grown in sterilized sea water in the light, and, further, that the concentration of the vitamin attained in these organisms is very high. Many other marine algae also contain this vitamin. These vegetable organisms form the food of a host of minute animals, which in their turn are eaten by many kinds of small fish, and these in theirs by the cod and other fish. Readers interested in this aspect of the matter may like to be referred to the article on "Photosynthesis and sea air," published in the British Medical Journal on April 7th, 1923 (p. 601).

Of peculiar interest is the effect of deprivation of this vitamin in causing liability to infection. Direct experiments show no change in the agglutinin and amboceptor titre or of the opsonic index of the serum of animals fed on diets deficient in the vitamin; there is evidence of remarkable diminution in blood platelets, but this observation at present lacks confirmation. Increased susceptibility to infection is, however, one of the most important of the effects of deprivation of this vitamin, although not so far demonstrable by means of the test tube or pipette.

Vitamin $B$.

Chapter IV is headed "Deficiency Diseases: Beri-beri: Vitamin B (water-soluble B, anti-neuritic vitamin)." In it beri-beri is dealt with and the probable identity of water-soluble $B$ and anti-neuritic (anti-beri-beri) factors are considered. Then follows an account of the distribution of the vitamin among foodstuffs, of experiments with pigeons and with rats, and of the use made of such experiments in determining the distribution of the vitamin in foodstuffs. The approximate relative values of the more important foodstuffis are given, and emphasis is laid on the concentration of this factor in the germs of seeds. The experiments with pigeons on which these approximations are based were of two orders-preventive and curative-the former being considered the more valuable. In the preventive experiments the birds were fed artificially, and an estimate was made of the daily ration of the particular foodstuff necessary to prevent polyneuritis in pigeons, when added to a vitamin-free diet consisting of highly milled and polished rice. In the absence of an adequate supply of vitamin in the supplementary foodstuff the birds developed severe symptoms in from fifteen to twenty-five days, with paralysis of legs and wings, leading to complete helplessness and death within twenty-four to forty-eight hours. Protection was reckoned to be successfully accomplished if the birds showed no symptoms after a period of fifty to sixty days. Objections might be raised to the results reached in these experiments were it not that they are definitely stated to be approximations. Nevertheless the comment may be made that polyneuritis columbarum may develop after periods as prolonged as 120 days, and that considerable variation exists among individual pigeons in regard to their susceptibility to deficiency of vitamin $\mathbf{B}$.

The properties of vitamin $\mathbf{B}$ are then discussed, and an account given of the attempts to isolate it. Its origin and physiological significance, its effect on the secretory power of glands, its relation to other constituents of the food, its storage in the animal organism, its excretion, and its relation to the growth of the higher plants, fungi, reasts, and bacteria, are all dealt with in due order. To peruse this important chapter is to realize the far-reaching importance of this remarkable food essential, and to believe that, much as we have learned of the part it plays in nutrition, we have as yet but touched the fringe of knowledge. The " anti-neuritic" label is still attached to it, and rightly so, perhaps, since it focuses attention on one of its functions; but its utility is wider than this, while it is still open to doubt whether want of this vitamin is indeed the sole factor responsible for the causation of beri-beri. It seems probable that its relation to gastrointestinal health is intimate, and that its deficiency in association with the other food faults which accompany it in human dietaries is concerned with much of the gastrointestinal ill health so common at the present day.

\section{Vitamin $C$.}

In Chapter V "Scurvy (Vitamin C, the Antiscorbutic Vitamin)" is discussed at length, and the same plan followed as in the discussion of vitamin $\mathbf{A}$ and vitamin $\mathbf{B}$ in the preceding chapters. A table showing the distribution of this factor in foodstuffs is given. One of the most important matters dealt with in this interesting chapter concerns the poverty of milk, in all its forms, in this essential factor and the necessity for its provision when infants are reared on cow's milk. The requirements of different species of animals for this vitamin are discussed, and here again we see the necessity for further knowledge in regard to the requirements of different individuals of the same species and of the factors which determine possible variations in this regard. In designing a dietary for human beings we have especially to cater for those that are most susceptible to deficiency of vitamins of whatever class, rather than for the average individual who can do with less of them. 


\section{Rickets.}

Chapter VI is lengthy; it deals with rickets as a deficiency disease. It is concluded that there are two outstanding factors involved in the etiology of rickets: (1) the amount of a substance (antirachitic vitamin) in the diet having similar physiological properties and distribution to ritamin $A$, and (2) the degree of exposure to sunlight. In addition stress is laid on the absolute and relative amounts of calcium and phosphorus in the diet, and on the amount and type of energy-bearing foodstuffs (especially cereals) in it. The interaction between sunlight, and other forms of light radiation, and food in the prevention and cure of rickets is discussed, and the hypothesis advanced that light radiation may be regarded either as an economizer of the antirachitic vitamin or as a mobilizer of reserve supplies in the tissues. Sunlight appears to make safe some diets that are relatively deficient in this vitamin.

This interaction between food and sunlight is thought to render intelligible the distribution of rickets in different races and in different communities. The question of the interaction between vitamins and other factors which may be concerned in the prevention or causation of disease is one of much importance, for it seems probable that, as in rickets, so in a number of other diseases, vitamin deficiency may be one of a set of factors concerned in their causation-but only one.

The succeeding chapters of the report (VII and VIII) are, for the practitioner of medicine, the most important, dealing as they do with the application of experimental work to the practical problems of human diets. They indicate in moderate terms how great is the importance of this work in therapeutics and how great the value both to man and animals of the experimental method of research. Already the results reached are capable of application in the prerention and treatment of disease; the capital expenditure entailed in the prosecution of the work already yields a high rate of interest. If criticism of chapters so admirable in their theme and execution be permissible at all, it is that, far as they go, they hardly go far enough. Yet it is well, perhaps, that a case resting on foundations so sure should be under rather than over stated.

Teeth: Pellagra.

The final chapter (IX) deals with the influence of diet on teeth. The discussion of pellagra, provisionally included in the first edition, has been omitted; the reason being that " while pellagra must still be regarded as a disease of dietetic origin, no fresh evidence has been brought forward in support of that view, and the latest researches upon the subject point to a quantitative or qualitative defect in the protein of the diet as playing the chief part in the etiology of this disease."

Conclusion.

The report as a whole should be studied by all who profess to heal the sick or to prevent disease, for whatever else in medical literature one may be forced to neglect in these days of much writing, one cannot afford to neglect this, nor to make the knowledge it provides part of one's mental equipment. Vitamins, in an otherwise properly constituted diet, are indispensable for proper metabolism; a thorough knowledge of them, in an otherwise properly constituted mental equipment, is indispensable for the proper practice of medicine. To quote the last paragraph of the introductory chapter: "The practical importance of the facts will not be understood unless it be recognized that a deficiency in food, which, when complete or extreme, leads to actual disease, may, when only relative, be responsible for ill health of a rague but still important kind."

The report is a milestone on the path of knowledge in regard to the relation of faulty food to disease; from it we can look back with satisfaction along the road already trarersed, and, turning, face the tedious journey ahead with courage, hope, and confidence.

R. McCarrison.

A PROFESSORSHIP of children's diseases has recently been established at the Strasbourg faculty of medicine, its first occupant being Dr. Rohmer, who has hitherto been in charge of the children's clinic at Strasbourg.

\section{fidita et Hetera.}

\section{THE SCAMNUM OF HIPPOCRATES.}

IN his work on the articulations Hippocrates described with considerable detail those methods which he had found the best for the reduction of dislocations of the hip-joint. Among other means what has been known as "the scamnum of Hippocrates" takes an important place. (Latin, scamnum, a bench or stool; from scando.) This was a table provided with means of extension and counter-extension; in short, it appears to have been the prototype of Hawley's and other tables which have been and are now used in the treatment of fractures and dislocations of the lower extremities. It is thus described in the English version of the Genuine Works of Hippocrates published by the Sydenham Society in 1849:

"It will be of importance for any person who practises medicine in a populous city to get prepared a quadrangular board, about six cubits or a little more in length, and about iwo cubits in breadth, a span [that is, nine inches]* will be sufficient thickness for it; and then along it from the one end to the other, an excavation must be made, so that the working of the levers may not be higher than is proper." [This is veryobscure.] "Then at both sides we are to raise short, strong and strongly fixed posts, having axles; and in the middle of the bench five or six long grooves are to be scooped out about four inches distant from one another, three inches will be a sufficient breadth for them and the depth in like manner; and although the number of grooves I have mentioned will be sufficient, there is nothing to prevent their being made all over the bench, and the bench should have in its middle a pretty deep hole, of a square shape, and of about three inches in size; and into this hole, when judged necessary, is to bo adjusted a corresponding piece of wood, round above, which at the proper time, is to be adjusted between the perineum and the head of the thigh-bone. This apright piece of wood prevents the body from yielding to the force dragging downwards by the feet; for sometimes this piece of wood serves the purpose of counter-extension upwards ; and sometimes, too, when extension and counter-extension are made, this piece of wood, if susceptible to some motion to this side or that, will serve the purpose of a lever for pushing the head of the thigh-bone outwards. It is on this account that several grooves are scooped out on the bench, so that this piece of wood, being erected at the one which answers, may act as a lever, either on the sides of the articular heads of bones, or may make pressure on the heads along with the extension, according as it may suit to push inwards or outwards with the lever, and the lever may be either a round or broad form as may be judged proper; for sometimes the one form and sometimes the other suits with the articulation. This mode of applying the lever along with extension is applicable in the reduction of all dislocations of the thigh. In the case now on hand, a round lever is proper; but in dislocations outwards a flat lever will be the suitable one. By means of such machines and of such powers, it appears to me that we need never fail in reducing any dislocation at a joint.

"And one might find out other modes of reduction for this joint. If the large bench were to have raised on it two posts about a foot [in diameter?], and of a suitable height, on each side near its middle, and if a transverse piece of wood like the step of a ladder, were inserted into the posts, then if the sound leg were carried through between the posts, and the injured limb were brought over the transverse piece of wood, which should be exactly adapted in height to the joint which is dislocated (and it is an easy matter so to adjust it, for the step of the ladder should be made a little higher than required, and a convenient robe, folded several times, is to be laid below the patient's body), then a piece of wood, of suitable breadth and length, is to be laid below the limb, and it should reach from beyond the ankle to the head of the thigh-bone, and should be bound moderately tight to the limb. Then the limb being extended, either by means of the pestle-like piece of wood (formerly described), or by any of the other methods of exten. sion, the limb which is carried over the step with the piece of wood attached to it, is to be forced downwards, while somebody grasps the patient above the hip-joint. In this manner the extension will carry the head of the thigh bone above the acetabulum, while the lever power that is exercised will push the head of the thigh-bone into its natural seat. All the abovementioned powers are strong, and more than sufficient to rectify the accident, if properly and skilfully applied. For, as formerly stated, in most cases reduction may be effected by much weaker extension, and an inferior apparatus."

*The Sydenham translation has "a fathom," which is absurd. In the original Greek the word is $\sigma \pi \imath \theta a \mu$ caio $\nu$ (dodrantalis) $=$ a span. 